\title{
Poder preditivo de uma escala de alerta precoce para deterioração clínica de pacientes críticos
}

Predictive power of an early warning scale for clinical deterioration of critically ill patients

Poder predictivo de una escala de alerta precoz para el deterioro clínico de pacientes críticos

\section{Juliana da Silva Garcia Nascimento ${ }^{\mathrm{I}}$, Gabriela de Oliveira Macedo ${ }^{\mathrm{II}}$, Giovanna Beirigo Borges ${ }^{\text {III }}$}

\begin{abstract}
Resumo: Objetivo: avaliar o poder preditivo de uma escala de alerta precoce modificada para identificação de deterioração clínica em pacientes críticos. Método: estudo descritivo, quantitativo, em hospital de ensino, com 214 indivíduos na unidade de terapia intensiva, por meio de revisão dos prontuários, de março a dezembro de 2018.

Resultados: o tempo médio de permanência foi de 10,42 dias e as doenças respiratórias consideradas a principal causa de admissão. Dentre a população estudada, 136 (63,6\%) obtiveram alta e 78 (36,4\%) foram a óbito. Dos 78 pacientes $(36,4 \%)$, a média do escore da escala de alerta precoce para deterioração na admissão foi de 3,410 e a média que antecedeu o óbito foi de 5,000. Conclusão: considerou-se a escala de alerta precoce utilizada neste estudo, um instrumento fidedigno para identificação da deterioração clínica, recomendando-a para prevenção de parada cardiorrespiratória em adultos no ambiente hospitalar.
\end{abstract}

Descritores: Índice de gravidade de doença; Unidades de terapia intensiva; Gravidade do paciente; Equipe de assistência ao paciente

\begin{abstract}
Aim: To evaluate the predictive power of a modified early warning scale to identify clinical deterioration in critically ill patients. Method: a descriptive, quantitative study in a teaching hospital with 214 individuals in the intensive care unit, through medical records review, from March to December 2018. Results: the average length of stay was 10.42 days and the respiratory diseases considered the main cause of admission. Among the population studied, $136(63.6 \%)$ were discharged and $78(36.4 \%)$ died. Of the 78 patients $(36.4 \%)$, the mean early warning scale score for admission deterioration was 3.410 and the mean before death was 5,000. Conclusion: the early warning scale used in this study was considered a reliable instrument to identify clinical deterioration, recommending it for the prevention of cardiorespiratory arrest in adults in the hospital environment.
\end{abstract}

Descriptors: Disease severity index; Intensive care units; Patient's severity; Patient Care Team

\footnotetext{
${ }^{\text {I }}$ Enfermeira. Mestre em enfermagem. Docente no Curso de Graduação em Enfermagem da Universidade de Uberaba. (UNIUBE), Uberaba, Minas Gerais, Brasil. E-mail: mestradounesp28@yahoo.com.br. ORCID: https://orcid.org/0000-0003-1118-2738

II Graduanda em Enfermagem. Universidade de Uberaba (UNIUBE), Uberaba, Minas Gerais, Brasil. E-mail: gabbyoliveiramacedo@hotmail.com. ORCID: https://orcid.org/0000-0001-5226-4399

III Enfermeira. Universidade de Uberaba (UNIUBE), Uberaba, Minas Gerais, Brasil. E-mail: giovannabeirigo@hotmail.com. ORCID: https://orcid.org/0000-0002-4777-958X
} 
Poder preditivo de uma escala de alerta precoce para deterioração clínica de pacientes críticos $\mid 2$

Resumen: Objetivo: evaluar el poder predictivo de una escala de alerta temprana modificada para identificar el deterioro clínico en pacientes críticos. Método: estudio descriptivo y cuantitativo en un hospital universitario con 214 personas en la unidad de cuidados intensivos, por revisión de registros médicos, de marzo a diciembre de 2018. Resultados: la duración promedio de la estadía fue de 10.42 días y el enfermedades respiratorias consideradas principal causa de ingreso. Entre la población estudiada, 136 (63.6\%) fueron dados de alta y 78 (36.4\%) murieron. De los 78 pacientes $(36,4 \%)$, la puntuación media de la escala de advertencia temprana para el deterioro de la admisión fue de 3.410 y la media antes de la muerte fue de 5.000. Conclusión: la escala de alerta utilizada en este estudio se consideró un instrumento confiable para identificar el deterioro clínico, recomendándolo para la prevención del paro cardiorrespiratorio en adultos en el entorno hospitalario.

Descriptores: Índice de gravedad de la enfermedad; Unidades de cuidados intensivos; Severidad del paciente; Equipo de atención al paciente

\section{Introdução}

Pacientes considerados graves ou potencialmente graves, são encaminhados para a internação em unidades hospitalares e demandam cuidados específicos, acurácia no atendimento e permanência em Unidades de Terapia Intensiva (UTI). ${ }^{1}$ Nessas unidades, eles podem apresentar, ao longo de sua internação, um estado de deterioração fisiológica caracterizado geralmente pela alteração de frequência cardíaca, frequência respiratória, pressão arterial, temperatura e nível de consciência. Se identificada precocemente, a deterioração fisiológica pode ser prevenida, impactando também, na diminuição da mortalidade hospitalar. ${ }^{2}$

Com o propósito de mensurar a qualidade e a eficácia do cuidado prestado aos pacientes graves durante o período de internação, surgiram, no final do século 20 , escalas que se propuseram cumprir estes objetivos. ${ }^{3}$ Escalas ou escores de gravidade pretendem oferecer recursos que estabeleçam um parâmetro para identificar a evolução clínica dos pacientes e apontar sinais que demonstrem instabilidade. ${ }^{2}$

Em 1997, no Reino Unido, desenvolveu-se a primeira escala para avaliação de gravidade clínica, composta por pontuações que mensuravam o nível de deterioração fisiológica de doentes, denominada Early Warning Score(EWS). ${ }^{4}$

Embasando-se na versão original do EWS, foi elaborada a escala Modified Early Warning Scoring (MEWS), instrumento de fácil manejo, executado à beira do leito, que objetiva 
3 | Nascimento JSG, Macedo GO, Borges GB

identificar rapidamente pacientes em estado alarmante e em risco de deterioração fisiológica catastrófica. ${ }^{4}$ Este instrumento possui valores compreendidos entre zero a 14 pontos, referentes ao nível de consciência, pressão arterial, frequência cardíaca, respiratória e temperatura corporal do paciente..$^{5-7}$

A literatura aponta que, em 79\% dos pacientes que apresentam parada cardiorrespiratória ocorreu anteriormente uma alteração importante dos sinais vitais, e que, destes, 54\% evoluem para o óbito, o que indica que, este desfecho clínico poderia ter sido evitado na maioria dos casos, no ambiente intra-hospitalar, principalmente pela utilização do MEWS pelos profissionais de saúde intra-hospitalar, fundamentais na identificação da deterioração fisiológica dos pacientes da implementação de ações preventivas. ${ }^{7-8}$ A utilização do MEWS para a identificação de deterioração fisiológica dos pacientes por enfermeiros, médicos, fisioterapeutas e técnicos em enfermagem, já em seu primeiro dia de internação em UTI, resulta em notável taxa de sobrevivência com melhora significativa da alta hospitalar, uma vez que estes profissionais tem a chance de planejar a assistência e antecipar cuidados. ${ }^{9-11}$

Mesmo diante da importância da adoção de escalas, como o MEWS, para detecção precoce de deterioração fisiológica, e do fato de que, o prognóstico dos pacientes admitidos em UTI tem relação direta com sua gravidade, a utilização desta escala é pouco explorada pela equipe de saúde e os estudos sobre sua eficácia são incipientes. ${ }^{9-12}$ Diante do exposto, o objetivo deste estudo foi avaliar o poder preditivo de uma escala de alerta precoce modificada para identificação de deterioração clínica em pacientes críticos.

\section{Método}

Trata-se de estudo descritivo, prospectivo, de abordagem quantitativa, realizado por meio de análise observacional, em um hospital universitário do Estado de Minas Gerais, destinado à assistência da população municipal e região com 220 leitos. A UTI adulto, especificamente, é 
Poder preditivo de uma escala de alerta precoce para deterioração clínica de pacientes críticos $\mid 4$

provida de estrutura assistencial especializada, constituída por 20 leitos, todos com monitores multiparamétricos, que possibilitam a avaliação da condição clínica com precisão, em tempo real. ${ }^{13}$

A população do estudo foi composta por 214 pacientes críticos da UTI, de acordo com o cálculo amostral estabelecido, devidamente realizado por profissional estatístico, que considerou a média de internações mensais desta população, com poder amostral de $80 \%$ e nível de significância de $5 \%$.

Foram incluídos pacientes clínicos, com idade maior ou igual a 18 anos e provindos do setor de urgência e emergência e enfermarias. Foram excluídos pacientes que estivessem sedados, cirúrgicos e que não possuíam familiares ou responsáveis presentes no momento de visita na UTI, para concordância quanto ao Termo de Consentimento Livre e Esclarecido.

A coleta de dados compreendeu o período de março a novembro 2018. Os dados clínicos utilizados para verificação do MEWS foram obtidos por revisão dos prontuários eletrônicos dos pacientes internados na UTI, priorizando dois momentos: a internação na UTI, para identificar o valor do MEWS na sua entrada no setor, e a última anotação dos parâmetros clínicos desejados, antes do desfecho do paciente, alta ou óbito.

Apesar da escala MEWS não ser ainda validada para o contexto nacional, considerou-se para sua adoção na presente pesquisa, a similaridade com o contexto evidenciado no estudo original de validação, ${ }^{14}$ e utilização em outros estudos brasileiros. ${ }^{4,8}$

As pesquisadoras estiveram presentes, cinco dias por semana na UTI, durante o período de coleta, revezando sua permanência, e estabelecendo 2 horas de avaliação por dia, configurando em média de 5 a 6 pacientes entre diagnósticos clínicos admitidos na UTI e avaliações dos respectivos desfechos.

Devido a utilização da escala MEWS não ser uma prática adotada na realidade da UTI referente a presente pesquisa, para cumprir o objetivo de avaliar o seu poder preditivo quanto a 
5 | Nascimento JSG, Macedo GO, Borges GB

deterioração fisiológica, as pesquisadoras elaboraram e validaram um instrumento em face e conteúdo, por meio da avaliação de três profissionais, doutores na área de urgência e emergência, com a devida expertise na temática proposta.

Este instrumento apresentou duas partes, a primeira abordou a caracterização dos indivíduos, contendo sexo, idade, principal sistema orgânico acometido, data de admissão na UTI e tempo de permanência neste setor, já a segunda indicou o registro de características clínicas como; nível de consciência, pressão arterial sistólica, frequência cardíaca, frequência respiratória e a temperatura corporal.

O paciente que obtém valor de MEWS igual a zero, não possui deterioração fisiológica e deve continuar monitorado e observado, já, a pontuação 14, indica deterioração fisiológica instalada e determina o encaminhamento para UTI. ${ }^{5-7}$ No entanto, o escore 3 , ou acima de 3 , já é considerado um alerta importante para a deterioração fisiológica do paciente. ${ }^{5-6}$ À medida que a pontuação atribuída pelo MEWS aumenta, a necessidade de cuidados urgentes também se intensifica, constatando a proporcionalidade entre eles. ${ }^{6}$

Desta forma, foi possível realizar a somatória dos valores correspondentes a cada variável presente na escala MEWS, para posterior avaliação do seu poder preditivo quanto à deterioração clínica dos pacientes graves.

Os dados foram organizados em planilhas do Microsoft Exce® ${ }^{\circledR}$ 2013, com dupla digitação por dois pesquisadores diferentes, e analisados no Statistical Package for the Social Sciences (SPSS). Para análise estatística, média, mediana, desvio padrão, valores mínimos e máximo foram calculados para variáveis quantitativas, enquanto a distribuição de frequências absolutas e relativas foi calculada para variáveis qualitativas. O teste $t$ pareado foi utilizado para comparar as médias do escore de alerta precoce modificado do momento de admissão dos pacientes com o momento que antecedeu o desfecho principal (o óbito), considerando nível de significância de $5 \%(\alpha=0,05)$. 
Poder preditivo de uma escala de alerta precoce para deterioração clínica de pacientes críticos 6

Este estudo foi encaminhado para o Comitê de Ética a fim de se cumprirem as exigências da Resolução 466/12 do Conselho Nacional de Saúde, recebendo o protocolo 2.424.370. Obtevese o Termo de Anuência da instituição hospitalar proposta quanto a autorização para acesso a unidade de terapia intensiva, realização da pesquisa e manipulação dos prontuários. Para participação no estudo, os familiares foram consultados quanto ao interesse e assinaram o Termo de Consentimento Livre e Esclarecido em duas vias, assim como os pacientes que no momento da abordagem, encontravam-se orientados, sendo informados de todas as peculiaridades da pesquisa.

\section{Resultados}

A presente pesquisa teve a intenção de avaliar o poder preditivo da escala MEWS quanto a ocorrência de deterioração fisiológica de pacientes internados na UTI. Desta forma, identificouse que a idade dos indivíduos apresenta uma média de 69,23 anos, variando entre 18 a 99 anos. O menor tempo de internação dos pacientes neste setor foi de 1 dia e o maior de 78 dias, sendo que, a média, de permanência, foi de 10,42 dias na UTI. A tabela 1 demonstra o perfil sociodemográfico dos pacientes incluídos neste estudo.

Tabela 1 - Caracterização dos pacientes de acordo com o sexo, principal sistema orgânico acometido e desfecho do paciente na Unidade de Terapia Intensiva. Uberaba (MG), Brasil, 2018.

\begin{tabular}{lcc}
\hline Variáveis & $\mathbf{n}$ & $\mathbf{\%}$ \\
\hline Sexo & 96 & 44,9 \\
Masculino & 118 & 55,1 \\
Feminino & & \\
& & \\
Principal Sistema Orgânico Afetado & 35 & 16,4 \\
Neurológico & 59 & 27,6 \\
Respiratório & 42 & 19,6 \\
Cardiovascular & 17 & 7,9 \\
Gastrintestinal & 16 & 7,5 \\
Renal & 6 & 2,8 \\
Hematológico & 1 & 0,5 \\
Endócrino & & \\
\hline
\end{tabular}


7 | Nascimento JSG, Macedo GO, Borges GB

\begin{tabular}{lcc}
\hline $\begin{array}{l}\text { Imunológico } \\
\text { Desfecho }\end{array}$ & 38 & 17,7 \\
Alta & 136 & \\
Óbito & 78 & 36,6 \\
\hline
\end{tabular}

A maioria era do sexo feminino, representando 118 pacientes $(55,1 \%)$. O acometimento do sistema respiratório $(27,6 \%)$ foi o motivo principal de admissão na UTI adulto, seguido do acometimento nos sistemas cardiovascular (19,6\%), imunológico $(17,7 \%)$ e neurológico (16,4\%).

Dos 214 pacientes, $136(63,6 \%)$ tiveram a alta da UTI e 78 pacientes $(36,4 \%)$ tiveram o óbito como desfecho nesta mesma circunstância. As tabelas 2 e 3 a seguir, demonstram respectivamente, o óbito como desfecho, evidenciando a interpretação do MEWS, e em seguida a alta e a respectiva média do MEWS para comparação.

Tabela 2 - Valores da Modified Early Warning Scoring (MEWS), referente a população que apresentou o óbito como desfecho na Unidade de Terapia Intensiva, $n=78$. Uberaba (MG), Brasil, 2018.

\begin{tabular}{ccccc}
\hline MEWS & Média & $\begin{array}{c}\text { Desvio } \\
\text { padrão }\end{array}$ & $\begin{array}{c}\text { Erro padrão } \\
\text { da média }\end{array}$ & Valor de p \\
Admissão & & 2,0667 & 0,2340 & \\
Óbito & 3,410 & 3,3342 & 0,3775 & 0,001 \\
Diferença entre admissão e óbito & $-1,5897$ & 3,7605 & 0,4258 & \\
\hline
\end{tabular}

Do total de 78 pacientes $(36,4 \%)$ que tiveram o óbito como desfecho na UTI, a média do MEWS na admissão foi de 3,410 e a média do valor de MEWS que antecedeu o óbito foi de 5,000, apresentando valor de $\mathrm{p}$ estatisticamente significativo para deterioração fisiológica dos pacientes $(0,001)$. 
Poder preditivo de uma escala de alerta precoce para deterioração clínica de pacientes críticos $\mid 8$

Tabela 3 - Valores da Modified Early Warning Scoring (MEWS), referente a população que apresentou a alta como desfecho na Unidade de Terapia Intensiva, $\mathrm{n}=136$. Uberaba (MG), Brasil, 2018.

\begin{tabular}{lcccc}
\hline MEWS & Média & Desvio padrão & $\begin{array}{c}\text { Erro padrão } \\
\text { da média }\end{array}$ & Valor de p \\
\hline Admissão & 2,9635 & 1,80036 &, 15382 & \\
Alta & 2,9781 & 2,51310 &, 21471 & 0,952 \\
Diferença entre admissão e alta &,- 0146 & 2,85169 &, 24364 & \\
\hline
\end{tabular}

Notou-se uma média do valor de MEWS menor que 3 para os pacientes que obtiveram a alta da UTI como desfecho no período avaliado da presente pesquisa.

\section{Discussão}

Pesquisas que apontam características sociodemográficas e epidemiológicas de pacientes internados em UTI auxiliam na consolidação de mudanças das estratégias de cuidado. Além disso, culminam na melhoria da gestão da unidade, seja do ponto de vista humano, estrutural, de processos, ou mesmo da gestão do cuidado. ${ }^{15}$

A presente pesquisa identificou média de idade de 69,23 anos na população estudada. Assim, assemelhando-se a um estudo epidemiológico, descritivo e retrospectivo, realizado com 695 pacientes na UTI adulto, apontando que, mais da metade das internações (51,2\%) foi de pessoas com idade entre 40 e 69 anos. $^{15}$

Quando comparado com a literatura, o tempo médio de permanência dos pacientes deste estudo, de 10,42 dias, foi maior do que em outras unidades de terapia intensiva, que destacam um período $\leq 6$ dias. Permanências significativamente mais curtas do que o esperado podem indicar a presença de esforços voltados para a diminuição de custos, por meio da alta prematura dos pacientes ou maior gravidade dos casos (alta mortalidade nos primeiros dias/ horas), e, de outro modo, tempos de permanência significativamente mais longos do que o esperado podem indicar deficiências e dificuldades para o cuidado prestado. ${ }^{16}$ 
Quanto ao sexo, o resultado da presente pesquisa, contradiz os achados da literatura, ao apontar que a maioria da população era do sexo feminino (55,1\%). As características demográficas presentes nas UTI geralmente convergem para predominância do sexo masculino, com valores maiores do que 50\% das internações em UTI. ${ }^{17}$

Esta contradição advém, possivelmente do maciço incentivo, na atualidade de estratégias preventivas, realizadas com a população masculina, para o controle de doenças específicas e à consequente conscientização e adesão, por parte dos homens, aos programas de saúde, o que vem minimizando os agravos e reduzindo sua internação em UTI. ${ }^{18-19}$

As doenças do trato respiratório constituíram a principal causa de internação, seguidas pelas cardiovasculares. Estes achados corroboram com os resultados de um estudo epidemiológico realizado com 171 pacientes, que destacou as doenças cardiorrespiratórias como principais motivos de internação na UTI. ${ }^{20}$

As demandas clínicas dos pacientes na UTI se sobressaem frente às cirúrgicas, possivelmente devido à agudização de problemas crônicos. Agravos clínicos de caráter agudo e idade acima de 80 anos estão associados à mortalidade nos estudos de sobrevida com acompanhamento menor que 30 dias, e situações como rebaixamento do nível de consciência, uso de ventilação mecânica e doenças respiratórias são fatores que pioram esse desfecho. ${ }^{20}$

Quanto ao desfecho clínico, a maioria dos pacientes deste estudo, teve alta do setor, apresentando uma média de MEWS menor que 3, o que evidencia ausência de deterioração fisiológica, ${ }^{21}$ porém um número considerável apresentou o óbito como desfecho. Geralmente, utiliza-se a gravidade das doenças para prever a mortalidade dos pacientes em UTI, identificando-se elevada taxa de mortalidade neste ambiente, que varia entre 5,4 e $33 \%$, de acordo com o cenário internacional. ${ }^{21}$ Porém, no âmbito nacional, a taxa de óbitos revela-se acima da internacional, de 36,9 a 50,74\%. ${ }^{22-24}$ 
Poder preditivo de uma escala de alerta precoce para deterioração clínica de pacientes críticos|

Os pacientes que apresentaram o óbito como desfecho clínico nesta pesquisa tiveram um valor médio de MEWS no momento da admissão de 3,410 e de 5,000 na última avaliação dos parâmetros clínicos, anterior ao óbito. Os resultados demonstraram diferença estatisticamente significativa na associação entre o valor do MEWS e o desfecho (mortalidade) dos pacientes na UTI, apresentando valor de p igual a 0,001.

Pacientes que obtiveram soma $\geq 5$ pontos no MEWS foram associados a maior risco de morte e admissão em UTI. ${ }^{25}$ Este fato reforça a necessidade do uso da escala MEWS pelos profissionais de saúde nos setores de emergência, nas enfermarias, para reconhecer precocemente a deterioração fisiológica, antes da admissão na UTI e para evitá-la. ${ }^{24}$

Esta escala tem a intencionalidade principal de facilitar a comunicação entre as equipes de saúde quanto à deterioração fisiológica do paciente e precavê-la. Nesse sentido, de modo a evitar a transferência do paciente para UTI e ainda, caso a transferência seja inevitável, garantir que ela ocorra sem demora., ${ }^{4,25}$

Pacientes admitidos em UTI têm variável morbimortalidade e geralmente apresentam sinais de alerta alguns dias antes de sua internação, ${ }^{8}$ o que é demonstrado, nesta pesquisa, pelo valor já elevado do escore de alerta precoce, identificado no momento da admissão nesta unidade (MEWS=3,410). Assim, evidenciando que, as alterações fisiológicas, que indicam a piora do estado clínico, podem denunciar precocemente os potenciais pacientes críticos, que necessitarão de monitorização especial nas enfermarias, ou salas de emergência. ${ }^{8}$

Quando o paciente é oriundo das enfermarias ou salas de emergência geralmente tem grande morbidade no momento da admissão na UTI, alertados pelo valor de MEWS maior do que 3 pontos em mais de $70 \%$ da população, com média superior a 5 pontos. ${ }^{8}$ Estes dados foram confirmados neste estudo, e apesar de demonstrar taxa de mortalidade menor do que a identificada no cenário nacional, ${ }^{23}$ apresenta forte associação entre a presença de escores 
11 | Nascimento JSG, Macedo GO, Borges GB

críticos ( $\geq 3$ pontos) já na admissão dos pacientes e sua deterioração fisiológica, com aumento significativa da média do MEWS para 5,000 apresentada pela maioria em seu desfecho.

Deve-se, portanto, haver incentivo para adoção da escala MEWS, no contexto intrahospitalar de cuidado a saúde. Isto, visto sua potencialidade para prever a deterioração fisiológica dos pacientes, evitar desfechos indesejáveis e irreversíveis, ser de fácil manuseio e interpretação dos profissionais de saúde. ${ }^{8}$

\section{Conclusão}

O valor de MEWS maior ou igual a 3 esteve relacionado com a deterioração clínica na UTI, configurando-se em um alerta de deterioração, já no momento da admissão dos pacientes. Houve relevância estatística significante quanto à associação do MEWS e o óbito na UTI, o que evidencia o poder preditivo desta escala para desfechos irreversíveis e indesejados.

A limitação caracterizou-se pelo fato de a coleta de dados ter se concentrado em apenas uma UTI, o que dificulta a generalização dos achados. A contribuição principal e a implicação para a prática é a demonstração da eficácia do MEWS quanto à identificação de deterioração fisiológicas de pacientes no ambiente intra-hospitalar, considerando sua importância para a tomada de decisão precoce e efetiva em relação aos pacientes, na intenção principal de viabilizar a prática baseada em evidências, para aperfeiçoamento de competência profissional e segurança dos pacientes.

Considera-se a escala de alerta precoce utilizada é um instrumento fidedigno e eficaz para identificar a deterioração fisiológica dos pacientes, recomendando-a para prevenção de parada cardiorrespiratória em adultos. 
Poder preditivo de uma escala de alerta precoce para deterioração clínica de pacientes críticos|

\section{Referências}

1. Dobkin C, Finkelstein A, Kluender R, Notowidigd MJ. The economic consequences of hospital admissions. Am Econ Rev [Internet]. 2018 [acesso em 2019 ago 23];108(2):308-52. Disponível em: https://doi.org/10.1257/aer.20161038

2. Miranda JO, Camargo CL, Nascimento Sobrinho CL, Portela DS, Monaghan A, Freitas KS, et al. Tradução e adaptação de um escore pediátrico de alerta precoce. Rev Bras Enferm [Internet]. 2016 [acesso em 2018 nov 12];69(5):833-41. Disponível em: http://dx.doi.org/10.1590/0034-7167-2015-0096

3. Silva LC, Nogueira LS, Settervall CH, Sousa RM, Padilha KG. Desempenho de índices de gravidade para estimar risco de morte em Unidades de Terapia Intensiva. Rev Esc Enferm USP. 2012;46(4):846-50.

4. Rocha TF, Neves JG, Viegas K. Escore de alerta precoce modificado: avaliação de pacientes traumáticos. Rev Bras Enferm [Internet]. 2016 [acesso em 2018 nov 12];69(5):850-5. Disponível em: http://dx.doi.org/10.1590/0034-7167-2015-0145

5. Kruisselbrink R, Kwizera A, Crowther M, Robichaud AF, O’Shea T, Nakibuuka J, et al. Modified Early Warning Score (MEWS) identifies critical illness among ward patients in a resource restricted setting in Kampala, Uganda: a prospective observational study. PLos ONE [Internet]. 2016 [acesso em 2018 abr 22];11(3):e0151408.

Disponível

em:

https://journals.plos.org/plosone/article?id=10.1371/journal.pone.0151408

6. Westphal GA, Lino AS. Rastreamento sistemático é a base do diagnóstico precoce da sepse grave e choque séptico. Rev Bras Ter Intensiva [Internet]. 2015 [acesso em 2018 abr 26];27(2):96-101. Disponível em: http://www.scielo.br/scielo.php?script=sci_arttext\&pid=S0103-507X2015000200096

7. Freitas $\mathrm{CM}$, Preto EP, Nascimento $\mathrm{CAF}$. Intervenções de enfermagem na monitorização da deterioração clínica da pessoa em enfermaria hospitalar: uma revisão integrativa. Rev Enferm Referência [Internet]. 2017 [acesso em 2019 ago 22];4(14):121-32. Disponível em: http://www.scielo.mec.pt/scielo.php?script=sci_abstract\&pid=S0874-02832017000300013\&lng=pt\&nrm=iso

8. Tavares RC, Vieira AS, Uchoa LV, Peixoto Júnior AA, Meneses FA. Validação de um escore de alerta precoce pré-admissão na unidade de terapia intensiva. Rev Bras Ter Intensiva. 2008;125(20):124-7.

9. Johnson S, Nileswar A. Effectiveness of Modified Early Warning Score (MEWS) in the outcome of inhospital adult cardiac arrests in a tertiary hospital. J Pulm Respir Med [Internet]. 2015 [acesso em 2018 abr 20];5:4. Disponível em: http://dx.doi.org/10.4172/2161-105X.1000285

10. Salottolo K, Carrick M, Johnson J, Gamber M, Bar-Or B. A retrospective cohort study of the utility of the modified early warning score for interfacility transfer of patients with traumatic injury. BMJ Open [Internet]. 2017 [acesso em 2018 nov 14];7(5):e016143. Disponível em: https://bmjopen.bmj.com/content/bmjopen/7/5/e016143.full.pdf 
11. Nishijima I, Domari SO, Domari S, Mae T, Risa I, Chisato K, et al. Características sociodemográficas e epidemiológicas de pacientes internados em uma Unidade de Terapia Intensiva. Rev Enferm UERJ. 2016;25:e7736.

12. Kyriacos U, Jelsma J, James M, Jordan S. Monitoring vital signs: development of a modified early warning scoring (MEWS) system for general wards in a developing country. PLoS One [Internet]. 2014 [acesso em 2018 out 11];9(1):e87073. Disponível em: https://www.ncbi.nlm.nih.gov/pubmed/24475226

13. Mário Palmério Hospital Universitário. Quem Somos [Internet]. Uberaba (MG); 2019 [acesso em 2019 maio 06]. Disponível em: http://www.hospitalmariopalmerio.uniube.br/quemSomos.php

14. Subbe CP, Kruger M, Rutherford P, Gemmel L. Validation of a modified early warnig score in medical admissions. QJM [Internet]. 2001 [acesso em 2018 ago 17];94(10):521-6. Disponível em: https://academic.oup.com/qjmed/article/94/10/521/1558977

15. Rodriguez AH, Bubi MB, Zandonadil OF, Zandonadill G, Rodriguezi MJ. Características epidemiológicas e causas de óbitos em pacientes internados em terapia intensiva. Rev Bras Enferm [Internet]. 2016 [acesso em 2018 nov 12];69(2):229-34. Disponível em: http://www.scielo.br/scielo.php?script=sci_arttext\&pid=S0034-71672016000200229\&lng=pt\&tlng=pt

16. Roque KE, Tonini T, Melo EC. Eventos adversos na unidade de terapia intensiva: impacto na mortalidade e no tempo de internação em um estudo prospectivo. Cad Saúde Pública [Internet]. 2016 [acesso em 2018 abr 12];32(10):e00081815. Disponível em: http://dx.doi.org/10.1590/0102-311X00081815

17. Perão OF, Bub MBC, Zandonadi GC, Martins MA. Características sociodemográficas e epidemiológicas de pacientes internados em uma Unidade de Terapia Intensiva de adultos. Rev Enferm UERJ [Internet]. 2017 [acesso em 2018 out 11];25:e7736. Disponível em: https://doi.org/10.12957/reuerj.2017.7736

18. Pereira SM, Lima FES, Gonçalves FIR, Silva NBS, Santos WQ, Sousa PV, et al. Acessibilidade dos homens a unidade básica de saúde. Rev Eletrônica Acervo Saúde [Internet]. 2019 [acesso em 2019 ago 23];11(2):e108. Disponível em: https://doi.org/10.25248/reas.e108.2019

19. Bibiano AMB, Moreira RS, Tenório MMGO, Silva VL. Fatores associados à utilização dos serviços de saúde por homens idosos: uma revisão sistemática da literatura. Ciênc Saúde Colet [Internet]. 2019 [acesso em 2019 ago 22];24(6):2263-78. Disponível em: http://www.cienciaesaudecoletiva.com.br/artigos/fatores-associados-a-utilizacao-dos-servicos-de-saudepor-homens-idosos-uma-revisao-sistematica-da-literatura/16407?id=16407

20. Silva LS, Mont'Alverne DG, Medeiros AI, Silva AG, Carvalho EM. Características dos pacientes sob assistência fisioterapêutica na UTI de um hospital universitário: estudo epidemiológico transversal. Rev Fisioter Saúde Funcional. 2015;5(1):50-8. 
21. Bonfada D, Santos MM, Lima KC, Garcia-Altés A. Análise de sobrevida de idosos internados em Unidades de Terapia Intensiva. Rev Bras Geriatr Gerontol [Internet]. 2017 [acesso em $2018 \mathrm{dez}$ 10];20(2):197-205. Disponível em: http://dx.doi.org/10.1590/1981-22562017020.160131

22. Hon KL, Luk MP, Fung WM, Li CY, Yeung KL, Liu PK, et al. Mortality, length of stay, bloodstream and respiratory viral infections in a pediatric intensive care unit. J Crit Care [Internet]. 2017 [acesso em 2018 abr 11];38:57-61. Disponível em: https://www.sciencedirect.com/science/article/pii/S0883944116305482. Epub 2016 set 30.

23. Gershengorn HB, Harrison DA, Garland A, Wilcox ME, Rowan KM, Wunsch H. Association of intensive care unit patient-to-intensivist ratios with hospital mortality. JAMA Intern Med [Internet]. 2017 [acesso em 2018 jun 05];177(3):388-96. Disponível em: https://jamanetwork.com/journals/jamainternalmedicine/fullarticle/2598511

24. Guia CM, Biondi RS, Sotero S, Lima AA, Almeida KJ, Amorim FF. Perfil epidemiológico e preditores de mortalidade de uma unidade de terapia intensiva geral de hospital público do Distrito Federal. Comun Ciênc Saúde. 2015;26(1-2):9-19.

25. Vieira AM, Parente EA, Oliveira LS, Queiroz AL, Bezerra ISM, Rocha HAL. Características de óbitos dos pacientes internados em uma unidade de terapia intensiva de hospital terciário. J Health Biol Sci [Internet]. 2019 [acesso em 2019 maio 12];7(1):26-31. Disponível em: http://docs.bvsalud.org/biblioref/2019/01/969718/5-1999.pdf

\section{Autor correspondente}

Nome: Juliana da Silva Garcia Nascimento

E-mail: mestradounesp28@yahoo.com.br

Endereço: Rua Osório Joaquim Guimarães, nº 281, Ap: 203, Bloco 05, Parque São Geraldo. Uberaba, Minas Gerais. Brasil.

CEP: $38030-130$

\section{Contribuições de Autoria}

\section{1 - Juliana da Silva Garcia Nascimento}

Concepção e planejamento do projeto de pesquisa, obtenção, análise e interpretação dos dados, redação e revisão crítica.

\section{2 - Gabriela de Oliveira Macedo}

Concepção e planejamento do projeto de pesquisa, obtenção, análise e interpretação dos dados, redação e revisão crítica.

3 - Giovanna Beirigo Borges 
15 | Nascimento JSG, Macedo GO, Borges GB

Concepção e planejamento do projeto de pesquisa, obtenção, análise e interpretação dos dados, redação e revisão crítica.

\section{Como citar este artigo}

Nascimento JSG, Macedo GO, Borges GB. Poder preditivo de uma escala de alerta precoce para deterioração clínica de pacientes críticos. Rev. Enferm. UFSM. 2019 [Acesso em: Anos Mês Dia]; vol.10 e5: 1-15. DOI:https://doi.org/10.5902/2179769238300 